\title{
Effect of arazyme on the lipopolysaccharide-induced inflammatory response in human endothelial cells
}

\author{
IN SIK KIM ${ }^{1}$, EUN JU YANG ${ }^{2}$, DONG-HA SHIN ${ }^{3}$, KWANG-HEE SON $^{4}$, HO-YONG PARK ${ }^{4}$ and JI-SOOK LEE ${ }^{5}$ \\ ${ }^{1}$ Department of Biomedical Laboratory Science, School of Medicine, Eulji University, Daejeon 301-746; \\ ${ }^{2}$ Department of Clinical Laboratory Science, College of Health and Therapy, Daegu Hanny University, Gyeongsan 712-715; \\ ${ }^{3}$ Insect Biotech Co., Ltd., Dajeon 305-811; ${ }^{4}$ Industrial Bio-Material Research Center, \\ Korea Research Institute of Bioscience and Biotechnology, Daejeon 305-806; \\ ${ }^{5}$ Department of Clinical Laboratory Science, Wonkwang Health Science University, \\ Iksan, Chonbuk 570-750, Republic of Korea
}

Received June 4, 2013; Accepted January 17, 2014

DOI: $10.3892 / \mathrm{mmr} .2014 .2231$

\begin{abstract}
Arazyme is a novel extracellular metalloprotease secreted by Aranicola proteolyticus. Endothelial cells are involved in the pathogenesis of a number of inflammatory diseases, induce uncontrolled cell viability and express various inflammatory mediators, including cytokines, chemokines, adhesion molecules and reactive oxygen species (ROS). In the current study, human umbilical vein endothelilal cells (HUVECs) were used to investigate the anti-inflammatory effects of arazyme following lipopolysaccharide (LPS) stimulation. Apoptosis of HUVECs due to LPS was inhibited by arazyme. In various inflammatory responses induced by LPS, arazyme inhibited the secretion of the monocyte chemoattractant protein-1 and interleukin-6, and the expression of vascular cell adhesion molecule-1 and intercellular adhesion molecule-1. Arazyme also suppressed ROS production in HUVECs. The action of arazyme was not associated with $\mathrm{NF}-\kappa \mathrm{B}$ activity in HUVECs. These results indicate that arazyme has anti-inflammatory properties in inflamed endothelial cells and may be useful as a therapeutic agent for inflammatory diseases associated with endothelial cells.
\end{abstract}

\section{Introduction}

Arazyme is a novel extracellular metalloprotease produced by Aranicola proteolyticus (also known as Serratia proteamaculans, an aerobic Gram negative symbiotic bacterium isolated from the intestine of the spider, Nephila clavata) $(1,2)$. A previous report demonstrated that arazyme has

Correspondence to: Dr Ji-Sook Lee, Department of Clinical Laboratory Science, Wonkwang Health Science University, 501 Iksandaero, Iksan, Chonbuk 570-750, Republic of Korea E-mail: jslee1216@wu.ac.kr

Key words: arazyme, endothelial cells, anti-inflammatory effect, lipopolysaccharide hepatoprotective activity against carbon tetrachloride-induced hepatic injury and that the mechanism involves increased expression of SMP30 and antioxidant proteins (3).

Human umbilical vein endothelial cells (HUVECs) are used in studies of various diseases, including angiogenesis, atherosclerosis and the inflammatory process. Notably, HUVECs are involved in the release of cytokines and chemokines, as well as cell migration during the inflammatory process $(4,5)$. Increased levels of vascular cell adhesion molecule-1 (VCAM-1) and intracellular adhesion molecule-1 (ICAM-1) are early markers of endothelial activation and dysfunction in inflammatory diseases (6). Activation of adhesion molecules on the surface of endothelial cells induces leukocyte migration and leads to inflammation (7). The HUVEC-associated inflammatory response is induced by a variety of inflammatory mediators, including lipopolysaccharide (LPS), tumor necrosis factor- $\alpha$ and oxidized-low density lipoprotein $(6,8,9)$. LPS is an integral part of the bacterial outer membrane and is a significant stimulator of inflammation. LPS induces the generation of reactive oxygen species (ROS) and upregulates cytokines, chemokines and adhesion molecules in HUVECs (5). These mechanisms contribute to endothelial dysfunction and may evoke endothelial cell-associated diseases. A number of studies have attempted to identify methods to protect against the inflammatory response and endothelial cell death (9-11). In the current study, the role of arazyme in the LPS-mediated inflammatory response in HUVECs was investigated.

\section{Materials and methods}

Reagents. Endothelial cell basal medium-2 (EBM-2), fetal bovine serum (FBS), recombinant human fibroblast growth factor (rhFGF), recombinant human epidermal growth factor (rhEGF), hydrocortisone, gentamicin sulfate, amphotericin-B (GA-1000), heparin, vascular endothelial growth factor (VEGF), ascorbic acid and long $\mathrm{R}$ insulin-like growth factor-1 ( $\left.\mathrm{R}^{3}-\mathrm{IGF}-1\right)$ were purchased from Lonza (Walkersville, MD, USA). Trypsin-EDTA was purchased from Life Technologies, Inc. (Gaithersburg, MD, USA). LPS was purchased from Sigma-Aldrich Korea (Seoul, Korea). 
2',7'-dichlorofluorescein diacetate (DCFDA) and Alexa Fluor 488 chicken anti-mouse IgG were purchased from Molecular Probes (Eugene, OR, USA). Normal rabbit IgG, anti-VCAM-1 and anti-ICAM-1 antibodies were purchased from Santa Cruz Biotechnology (Santa Cruz, CA, USA).

Enzyme purification. Arazyme was purified as described previously (2). Briefly, extracellular fractions were collected by centrifugation of the culture medium or by filtration using a $0.2-\mu \mathrm{m}$ membrane filter (Pall Life Sciences, Port Washington, NY, USA). Chromatography was performed on a DEAE-cellulose column equilibrated with $50 \mathrm{mM}$ potassium phosphate buffer ( $\mathrm{pH}$ 7.6). Bound proteins were eluted with a 0.1-0.5 $\mathrm{M}$ sodium chloride gradient at a flow rate of $400 \mathrm{ml} / \mathrm{h}$ and each fraction was concentrated with a $10 \mathrm{kD}$ cassette membrane (Pall Life Sciences). The protein solution was then loaded at a flow rate of $20 \mathrm{ml} / \mathrm{h}$ onto a Sephadex G-75 column (GE Healthcare Life Sciences, Pittsburgh, PA, USA) equilibrated previously with $50 \mathrm{mM}$ potassium phosphate buffer ( $\mathrm{pH}$ 7.8). Fractions containing proteolytic activity were concentrated with the $10 \mathrm{kD}$ cassette membrane and stored at $-20^{\circ} \mathrm{C}$.

Cell culture. HUVECs were purchased from the American Type Culture Collection (Manassas, VA, USA). The cells were cultured on $0.2 \%$ gelatin-coated flasks with EBM-2 medium supplemented with $2 \%$ FBS, rhFGF, rhEGF, hydrocortisone, GA-1000, heparin, VEGF, ascorbic acid and R3-IGF-1. HUVECs were incubated at $37^{\circ} \mathrm{C}$ in a $5 \% \mathrm{CO}_{2}$ incubator.

MTT assay. The MTT assay was performed with a cell proliferation kit (Roche Diagnostics, Mannheim, Germany) to determine cell viability. HUVECs at a concentration of $5 \times 10^{3}$ cells/100 $\mu \mathrm{l}$ were plated on a 96-well plate. Following arazyme treatment, the plate was incubated for $24 \mathrm{~h}$ at $37^{\circ} \mathrm{C}$ in a $5 \% \mathrm{CO}_{2}$ incubator. Next, $10 \mu \mathrm{l}$ MTT solution was added to each well and the plate was incubated at $37^{\circ} \mathrm{C}$ for $4 \mathrm{~h}$ in a $\mathrm{CO}_{2}$ incubator. A $100 \mu \mathrm{l}$ aliquot of solubilization solution was added to each well. Following $24 \mathrm{~h}$ incubation, the absorbance was measured at $550 \mathrm{~nm}$ using an enzyme-linked immunosorbent assay (ELISA) reader (Bio-Tek Instruments Inc., Winooski, VT, USA).

Cell apoptosis. An Annexin V-fluorescein isothiocyanate (FITC) Apoptosis Detection kit (BD Biosciences, San Jose, CA, USA) was used to detect apoptosis. Following a $24 \mathrm{~h}$ treatment with stimulators, including LPS and arazyme, HUVECs were incubated with the FITC-labeled Annexin V and propidium iodide (PI) for $15 \mathrm{~min}$ at room temperature. Apoptotic cells were analyzed by flow cytometry using CellQuest software (BD Biosciences) and were defined as cells in the right quadrant, which stained positive for Annexin $\mathrm{V}$ with/without PI. A total of 10,000 events were collected for each sample.

ELISA. HUVECs were treated with LPS for $24 \mathrm{~h}$ and the supernatants were collected. The concentrations of monocyte chemoattractant protein-1 (MCP-1) and interleukin-6 (IL-6) were measured in the cell supernatant with a sandwich ELISA (OptEIA ${ }^{\mathrm{TM}}$ Set human IL-6 and MCP-1; BD Biosciences) according to the manufacturer's instructions. The concentration of each protein was calculated from standard curves.

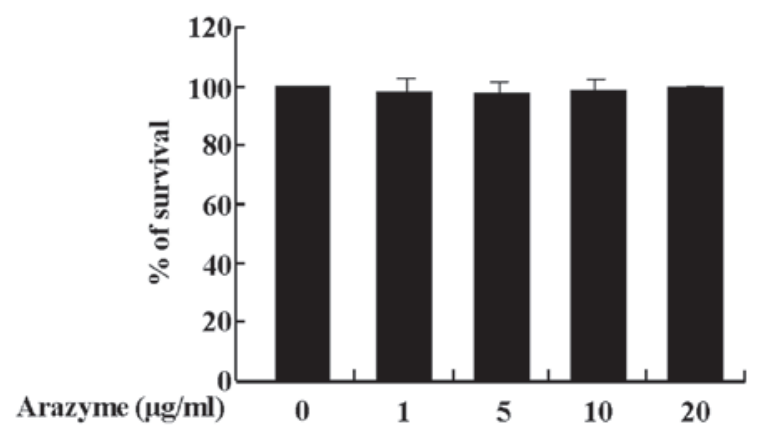

Figure 1. Arazyme has no cytotoxic effect on HUVECs. HUVECs seeded into 96 -well plates at $5 \times 10^{3}$ cells/well were cultured in the appropriate medium and were treated with arazyme in a dose-dependent manner for $24 \mathrm{~h}$ Survival rate was measured by an MTT assay. Data are expressed as the relative ratio to the absorbance of untreated cells, which was set to $100 \%$. All data are expressed as the mean \pm standard error of the mean of three independent experiments. HUVECs, human umbilical vein endothelilal cells.

VCAM-1 and ICAM-1 expression. To detect the surface expression of adhesion molecules, including VCAM-1 and ICAM-1, HUVECs were treated with LPS for $24 \mathrm{~h}$, incubated with anti-VCAM-1 and anti-ICAM-1 or control IgG antibodies for $30 \mathrm{~min}$ and then with anti-mouse IgG FITC-conjugated antibodies. The samples were analyzed with CellQuest software on a FACSCalibur flow cytometer (BD Biosciences). A total of 10,000 events was collected for each experiment.

Nuclear factor (NF)- $\kappa B$ p65 transcription factor assay. Following a $4 \mathrm{~h}$ stimulation with LPS, the DNA-binding activity of NF- $\kappa B$ in HUVECs was assessed using EZ-Detect ${ }^{\mathrm{TM}}$ Transcription Factor kits for NF- $\kappa B$ p65 (Pierce Biotechnology Inc., Rockford, IL, USA), following the manufacturer's instructions. DNA binding specificity was assessed using wild type or mutant NF- $\kappa \mathrm{B}$ oligonucleotides. Chemiluminescent detection was performed using a luminometer (Thermo Fisher Scientific, Pittsburgh, PA, USA).

ROS production. Following 24 h LPS stimulation, HUVECs were washed and resuspended at a concentration of $1 \times 10^{6}$ cells $/ \mathrm{ml}$ in pre-warmed PBS. The cells were exposed to $5 \mu \mathrm{M}$ DCFDA to label intracellular ROS and then incubated for $10 \mathrm{~min}$ at room temperature. Labeled cells were immediately observed by flow cytometry (BD Biosciences).

Statistical analysis. All data are expressed as the mean \pm standard error of the mean. Data were analyzed with Student's t-test and the SPSS statistical software package, version 10.0 (SPSS Inc., Chicago, IL, USA). $\mathrm{P}<0.05$ was considered to indicate a statistically significant difference.

\section{Results}

Arazyme inhibits HUVEC apoptosis induced by LPS. Prior to examining the effect of arazyme on HUVECs, the effect of arazyme on cell viability was examined. As shown in Fig. 1, the survival rate of HUVECs was not altered by arazyme treatment at concentrations of $1,5,10$ or $20 \mu \mathrm{g} / \mathrm{ml}$. Thus, $20 \mu \mathrm{g} / \mathrm{ml}$ arazyme was used to determine the anti-inflammatory effects. Since LPS causes endothelial injury by inducing apoptosis, the 

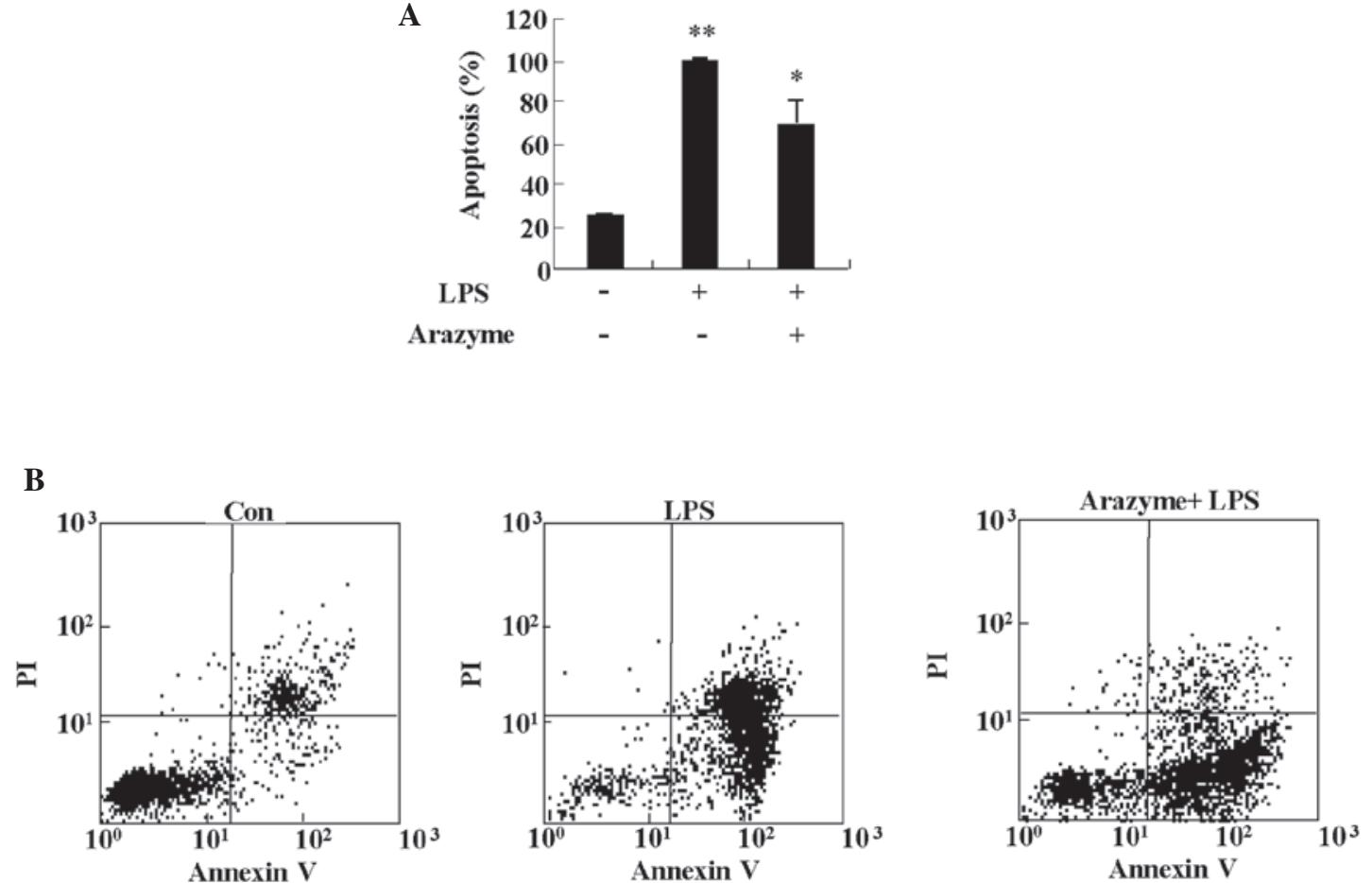

Figure 2. Arazyme inhibits LPS-induced HUVEC apoptosis. HUVECs were pre-treated with $20 \mu \mathrm{g} / \mathrm{ml}$ arazyme for $1 \mathrm{~h}$ and with $10 \mu \mathrm{g} / \mathrm{ml} \mathrm{LPS} \mathrm{for} 24 \mathrm{~h}$. Apoptosis was analyzed by measuring the binding of Annexin V-fluorescein isothiocyanate and PI by flow cytometry. The con group included cells without LPS treatment and was the negative control. The percentage of apoptotic cells represent the percentage of all Annexin-V binding cells in the total population. Data are expressed as the mean \pm standard error of the mean of (A) three individual experiments and are presented as (B) dot plot data. ${ }^{*} \mathrm{P}<0.05$ and ${ }^{* *} \mathrm{P}<0.01$, the untreated group vs. the LPS-treated group or the LPS-treated group vs. the arazyme-treated group. mHUVECs, human umbilical vein endothelilal cells; LPS, lipopolysaccharide; PI, propidium iodide; con, control.
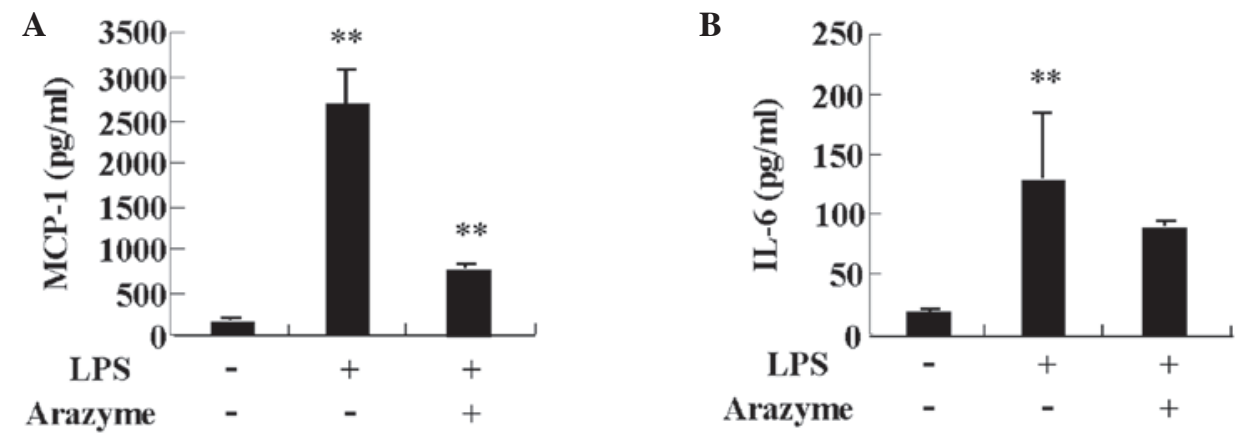

Figure 3. Arazyme has inhibitory effects on the release of LPS-induced MCP-1 and IL-6 from HUVECs. HUVECs were pre-treated in the absence or presence of $20 \mu \mathrm{g} / \mathrm{ml}$ arazyme for $1 \mathrm{~h}$. The cells were treated with $10 \mu \mathrm{g} / \mathrm{ml} \mathrm{LPS} \mathrm{for} 24 \mathrm{~h}$, the supernatants were collected and (A) MCP-1 and (B) IL-6 were analyzed by enzyme-linked immunosorbent assay. All data are expressed as the mean \pm standard error of the mean of three independent experiments. ${ }^{* *} \mathrm{P}<0.01$, the untreated group vs. the LPS-treated group or the LPS-treated group vs. the arazyme-treated group. LPS, lipopolysaccharide; MCP-1, monocyte chemoattractant protein-1; IL-6, interleukin-6; HUVECs, human umbilical vein endothelilal cells.

protective effect of arazyme on LPS-induced apoptosis was determined (12). As shown in Fig. 2, treatment with $10 \mu \mathrm{g} / \mathrm{ml}$ LPS led to strong induction of apoptosis in HUVECs. Arazyme significantly blocked LPS-induced apoptosis of HUVECs.

Arazyme decreases secretion of monocyte chemoattractant protein-1 (MCP-1) and IL-6 in HUVECs. To determine the inhibitory effect of arazyme on cytokine release from HUVECs, MCP-1 and IL-6 levels were measured by ELISA in HUVEC supernatants following treatment with $10 \mu \mathrm{g} / \mathrm{ml} \mathrm{LPS}$ in the presence or absence of arazyme. Arazyme decreased MCP-1 secretion considerably in HUVECs stimulated with
LPS (Fig. 3A). The release of IL-6 in LPS-stimulated HUVECs tended to be inhibited by arazyme, but the difference was not significant (Fig. 3B). These observations indicate that arazyme has anti-inflammatory effects on LPS-stimulated HUVECs by suppressing cytokine release.

Arazyme inhibits expression of adhesion molecules in HUVECs. LPS increases cell adhesion molecules, including VCAM-1 and ICAM-1 in HUVECs $(13,14)$. To determine other anti-inflammatory effects of arazyme, its effect on VCAM-1 and ICAM-1 expression induced by LPS was investigated. HUVECs were pretreated with $10 \mu \mathrm{g} / \mathrm{ml}$ arazyme for $1 \mathrm{~h}$ and 
A

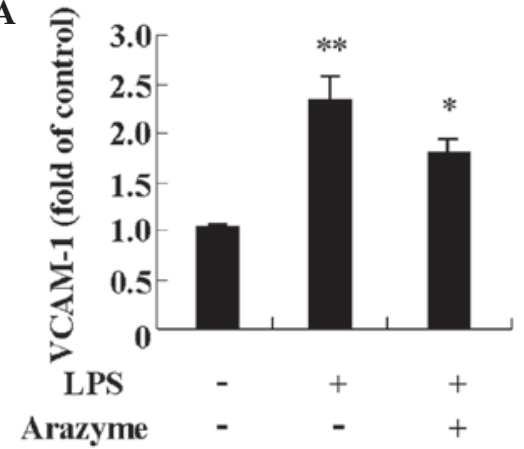

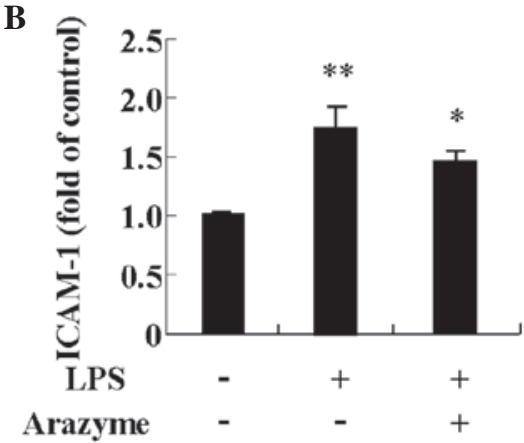

Figure 4. Arazyme suppresses the expression of adhesion molecules induced by LPS in HUVECs. HUVECs were pre-treated with $20 \mu \mathrm{g} / \mathrm{ml}$ arazyme for $1 \mathrm{~h}$ and then with $10 \mu \mathrm{l} / \mathrm{ml}$ LPS for $24 \mathrm{~h}$. Protein expression of (A) VCAM-1 and (B) ICAM-1 in HUVECs was analyzed by flow cytometry using anti-VCAM-1 and anti-ICAM-1 antibodies. The isotype con was analyzed by incubating with normal mouse IgG. Data are presented in relation to the isotype control, which was set to $100 \%$. ${ }^{*} \mathrm{P}<0.05$ and ${ }^{* *} \mathrm{P}<0.01$, the untreated group vs. the LPS-treated group or the LPS-treated group vs. the arazyme-treated group. HUVECs, human umbilical vein endothelilal cells; LPS, lipopolysaccharide; con, control; VCAM-1, vascular cell adhesion molecule-1; ICAM-1, intracellular adhesion molecule-1.

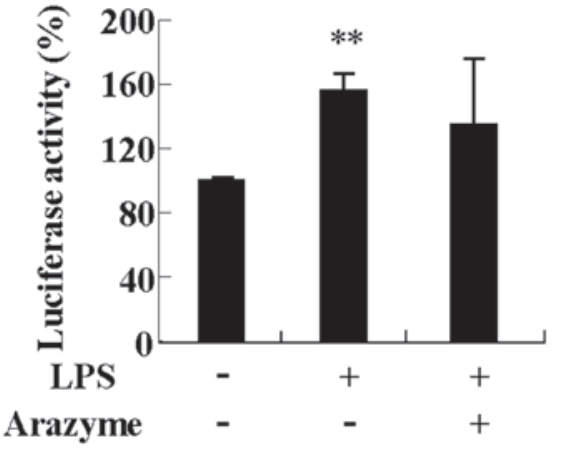

Figure 5. Arazyme is not involved in NF- $\kappa \mathrm{B}$ activation in HUVECs Following HUVEC pre-treatment with arazyme for $1 \mathrm{~h}$, the cells were treated with $10 \mu \mathrm{g} / \mathrm{ml}$ LPS for $24 \mathrm{~h}$ and collected to determine NF- $\kappa B$ activation. The nuclear fraction was extracted and NF- $\mathrm{KB}$ DNA binding activity was assessed using an EZ-Detect ${ }^{\mathrm{TM}}$ Transcription Factor kit. All data are expressed as the mean \pm standard error of the mean of three independent experiments. ${ }^{* *} \mathrm{P}<0.01$, the untreated group vs. the LPS-treated group or the LPS-treated group vs. the arazyme-treated group. NF- $\mathrm{kB}$, nuclear factor- $\mathrm{\kappa B}$; HUVECs, human umbilical vein endothelilal cells; LPS, lipopolysaccharide.

were then treated with $10 \mu \mathrm{g} / \mathrm{ml}$ LPS for $24 \mathrm{~h}$. LPS increased surface expression of VCAM-1 and ICAM-1 and the increased expression was suppressed by arazyme pretreatment (Fig. 4).

Arazyme is not associated with $N F-\kappa B$ activation induced by $L P S$ in HUVECs. NF- $\mathrm{KB}$ is a pleiotropic regulator of various genes and is involved in the inflammatory response, including the expression of chemokines, cytokines and adhesion molecules in HUVECs (15). Since LPS increases the expression of inflammatory proteins by activating NF- $\mathrm{KB}$, it was investigated whether the inhibitory effect of arazyme on cytokine and adhesion molecule expression was involved in inhibiting NF- $\kappa B$ activation. As shown in Fig. 5, arazyme had no effect on NF- $\kappa B$ activation induced by LPS in HUVECs. This result indicates that arazyme does not modulate the inflammatory response in HUVECs.

Arazyme inhibits LPS-induced ROS production in HUVECs. As ROS production is an important mediator of inflammation, the inhibitory effect of arazyme on ROS production in

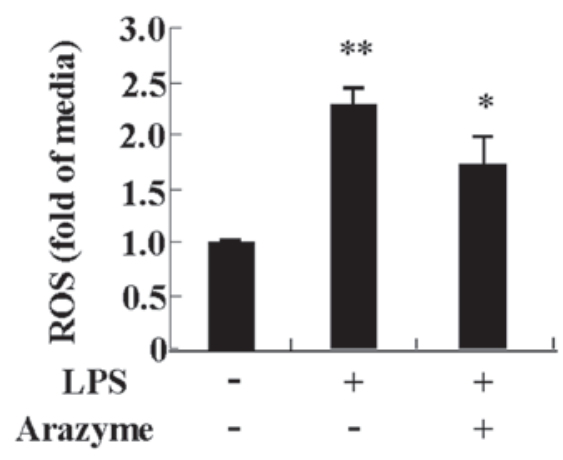

Figure 6. Arazyme reduces ROS production in LPS-stimulated HUVECs. HUVECs were pretreated with $20 \mu \mathrm{g} / \mathrm{ml}$ arazyme for $1 \mathrm{~h}$ and then with $10 \mu \mathrm{g} / \mathrm{ml}$ LPS for $4 \mathrm{~h}$. Following LPS stimulation, the HUVECs were incubated for $10 \mathrm{~min}$ with phosphate-buffered saline containing $3.3 \mu \mathrm{M}$ 2',7'-dichlorofluorescein diacetate and observed by flow cytometry analysis. The results are expressed as the mean \pm standard error of the mean of ten independent experiments. ${ }^{*} \mathrm{P}<0.05$ and ${ }^{* *} \mathrm{P}<0.01$, the untreated group vs. the LPS-treated group or the LPS-treated group vs. the arazyme-treated group. ROS, reactive oxygen species; HUVECs, human umbilical vein endothelilal cells; LPS, lipopolysaccharide.

HUVECs was examined. As shown in Fig. 6, LPS functioned as a strong inducer of ROS production in HUVECs. Pre-incubating HUVECs with arazyme suppressed ROS generation due to LPS. This result indicates that arazyme produces an anti-inflammatory effect by suppressing oxidative stress.

\section{Discussion}

Although arazyme has a protective effect against hepatic injury, other protective and therapeutic functions, including inflammation, have not yet been reported (3). Previous studies have identified novel inhibitory materials against the inflammatory response in endothelial cells such as Ecklonia cava extracts and cilostazol $(9,11)$. The current study, focused on elucidating the effect of arazyme on the inflammatory response in HUVECs induced by LPS. Cytokine release is an important inflammatory response. Arazyme inhibited MCP-1 and IL-6 secretion but to different degrees. MCP-1 is a CCL2 and acts as a chemotactic factor attracting monocytes (16). A previous study demonstrated that MCP-1 induces neutrophilic 
inflammation by inhibiting spontaneous apoptosis in neutrophils (17). IL-6 is a multipotent cytokine and increases the proliferation and differentiation of numerous cell types, including B cells and skin cells (18). IL-6 induces a shift from acute- to chronic-phase inflammation, including that observed in allergic diseases $(19,20)$. Arazyme also inhibited the expression of VCAM-1 and ICAM-1 increased by LPS. VCAM-1 and ICAM-1 function as adhesion molecules and initiators of the inflammatory signaling pathway (5). These results indicate that arazyme acts as an anti-inflammatory agent in HUVECs. ROS are generated at sites of inflammation and cause cellular injury and death (4). HUVECs modulate the movement of macromolecules and circulating immune cells from the blood into tissue. Increased oxidative stress in HUVECs induces vascular endothelial permeability and enhances leukocyte adhesion. Since arazyme suppresses ROS production due to LPS, arazyme may act as an inhibitor of inflammatory cell migration into tissue. Since hyperproduction of ROS induces cell death mediated by cytotoxicity, the anti-apoptotic effect of arazyme was hypothesized in HUVECs (21). The experimental results may indicate the validity of this hypothesis, despite the lack of a direct association between ROS and cell death.

A variety of inflammatory mediators, including LPS and ROS activate NF- $\mathrm{NB}$ and then induce an increase in adhesion molecules and the secretion of cytokines and chemokines (21). To examine the arazyme mechanism, NF- $\kappa \mathrm{B}$ activation was evaluated. However, arazyme did not inhibit NF- $\kappa \mathrm{B}$ activation caused by LPS stimulation. Although the mechanism underlying the effects of arazyme has not been previously determined, a hypothetical mechanism for the anti-apoptotic and anti-inflammatory effects may be suggested since arazyme is a metalloprotease. Arazyme may mediate its anti-apoptotic or anti-inflammatory functions via the protease-activated receptor (PAR), which is a G-protein-coupled receptor or through an unidentified receptor (22). PAR or an unknown-mediated signal, may be associated with inhibiting the LPS-induced signal. Secondly, arazyme may directly cleave cytokines, including MCP-1 and IL-6 and adhesion molecules, since it may hydrolyze pro-inflammatory molecules, including bradykinin and histamine $(23,24)$. The exact mechanism of action of arazyme remains to be determined and further studies are being conducted to investigate more complex mechanisms.

In conclusion, arazyme has anti-inflammatory effects in HUVECs that include suppression of cell apoptosis, ROS production, and expression of IL-6, MCP-1, VCAM-1 and ICAM-1. Arazyme may be a useful agent to treat endothelial dysfunction-associated diseases, including atherosclerosis and cardiovascular diseases.

\section{Acknowledgements}

This study was supported by a grant from the Korea Research Institute of Bioscience and Biotechnology Research Initiative Program.

\section{References}

1. Bersanetti PA,Park HY,Bae KS, Son KH,Shin DH,HirataIY,Juliano MA, Carmona AK and Juliano L: Characterization of arazyme, an exocellular metalloprotease isolated from Serratia proteamaculans culture medium. Enzyme Microb Technol 37: 574-581, 2005.
2. Kwak J, Lee K, Shin DH, Maeng JS, Park DS, Oh HW, Son KH, Bae KS and Park HY: Biochemical and genetic characterization of arazyme, an extracellular metalloprotease produced from Serratia proteamaculans HY-3. J Microbiol Biotechnol 17: 761-768, 2007.

3. Park JK, Jeong DH, Park HY, Son KH, Shin DH, Do SH, Yang HJ, Yuan DW, Hong IH, Goo MJ, et al: Hepatoprotective effect of arazyme on $\mathrm{CCl}_{4}$-induced acute hepatic injury in SMP30 knock-out mice. Toxicology 246: 132-142, 2008.

4. Hadi HA, Carr CS and Al Suwaidi J: Endothelial dysfunction: cardiovascular risk factors, therapy, and outcome. Vasc Health Risk Manag 1: 183-198, 2005.

5. Liu HT, He JL, Li WM, Yang Z, Wang YZ, Yin J, Du YG and $\mathrm{Yu} \mathrm{C}$ : Geniposide inhibits interleukin-6 and interleukin-8 production in lipopolysaccharide-induced human umbilical vein endothelial cells by blocking p38 and ERK1/2 signaling pathways. Inflamm Res 59: 451-461, 2010.

6. Szmitko PE, Wang CH, Weisel RD, Jeffries GA, Anderson TJ and Verma S: Biomarkers of vascular disease linking inflammation to endothelial activation: Part II. Circulation 108: 2041-2048, 2003.

7. Sprague AH and Khalil RA: Inflammatory cytokines in vascular dysfunction and vascular disease. Biochem Pharmacol 78: 539-552, 2009.

8. de Vries IJ, Langeveld-Wildschut EG, van Reijsen FC, Dubois GR, van den Hoek JA, Bihari IC, van Wichen D, de Weger RA, Knol EF, Thepen T and Bruijnzeel-Koomen CA: Adhesion molecule expression on skin endothelia in atopic dermatitis: effects of TNF-alpha and IL-4. J Allergy Clin Immunol 102: 461-468, 1998.

9. Kim TH and Bae JS: Ecklonia cava extracts inhibit lipopolysaccharide induced inflammatory responses in human endothelial cells. Food Chem Toxicol 48: 1682-1687, 2010.

10. Juzyszyn Z, Czerny B, Pawlik A and Droździk M: The effect of Artichoke (Cynara scolymus L.) extract on ROS generatin in HUVEC cells. Phytother Res 22: 1159-1161, 2008.

11. Lim JH, Woo JS and Shin YW: Cilostazol protects endothelial cells against lipopolysaccharide-induced apoptosis through ERK1/2- and P38 MAPK-dependent pathways. Korean J Intern Med 24: 113-122, 2009.

12. Munshi N, Fernandis AZ, Cherla RP, Park IW and Ganju RK: Lipopolysaccharide-induced apoptosis of endothelial cells and its inhibition by vascular endothelial growth factor. J Immunol 168: 5860-5866, 2002.

13. Marui N, Offermann MK, Swerlick R, Kunsch C, Rosen CA, Ahmad M, Alexander RW and Medford RM: Vascular cell adhesion molecule-1 (VACM-1) gene transcription and expression are regulated through an antioxidant-sensitive mechanism in human vascular endothelial cells. J Clin Invest 92: 1866-1874, 1993.

14. Sawa Y, Ueki T, Hata M, Iwasawa K, Tsuruga E, Kojima H, Ishikawa $\mathrm{H}$ and Yoshida S: LPS-induced IL-6, IL-8, VCAM-1, and ICAM-1 expression in human lymphatic endothelium. J Histochem Cytochem 56: 97-109, 2008.

15. Joyce DE and Grinnell BW: Recombinant human activated protein $\mathrm{C}$ attenuates the inflammatory response in endothelium and monocytes by modulating nuclear factor-kappaB. Crit care med 20 (Suppl 5): S288-S293, 2002.

16. Rossi D and Zlotnik A: The biology of chemokines and their receptors. Annu Rev Immunol 18: 217-242, 2000.

17. Yang EJ, Choi E, Ko J, Kim DH, Lee JS and Kim IS: Differential effect of CCL2 on constitutive neutrophil apoptosis between normal and asthmatic subjects. J Cell Physiol 227: 2567-2577, 2012.

18. Hirano T: Interleukin 6 and its receptor: ten years later. Int Rev Immunol 16: 249-284, 1998.

19. Gabay C: Interleukin-6 and chronic inflammation. Arthritis Res Ther 8 (Suppl 2): S3, 2006.

20. Bellanti JA: Cytokines and allergic diseases: clinical aspects. Allergy Asthma Proc 19: 337-341, 1998.

21. Viatour P, Merville MP, Bours V and Chariot A: Phosphorylation of NF-kappaB and IkappaB proteins: implications in cancer and inflammation. Trends Biochem Sci 30: 43-52, 2005.

22. Arora P, Ricks TK and Trejo J: Protease-activated receptor signalling, endocytic sorting and dysregulation in cancer. J Cell Sci 120: 921-928, 2007.

23. Hauck G: Proceedings: Vitalmicroscopic investigations of the effects of thrombin, a snake venom enzyme and histamine effect on the mesenteric microvasculature of rabbit and cat. Arzneimittelforschung 26: 1233, 1976.

24. Wolz RL and Bond JS: Phe5(4-nitro)-bradykinin: a chromogenic substrate for assay and kinetics of the metalloendopeptidase meprin. Anal Biochem 191: 314-320, 1990. 